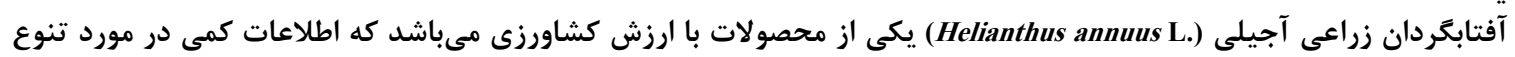

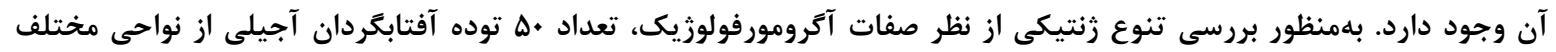

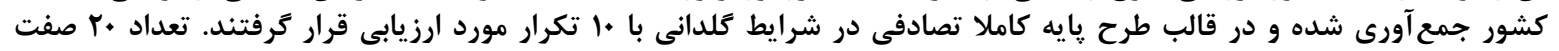

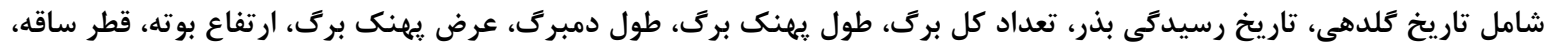

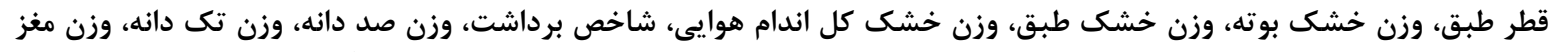

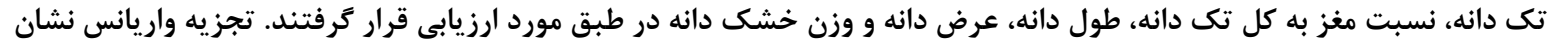

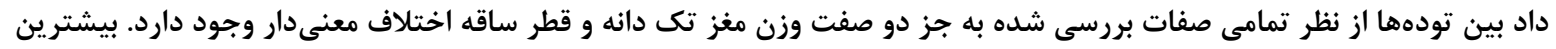

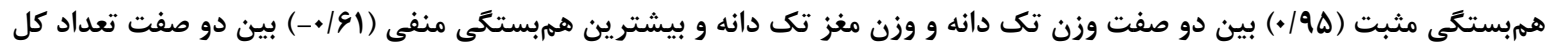

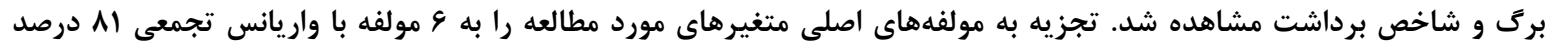

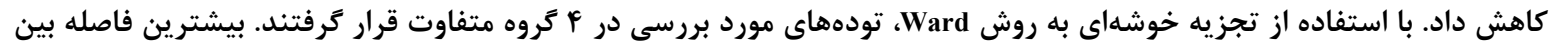

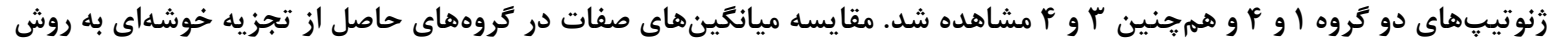

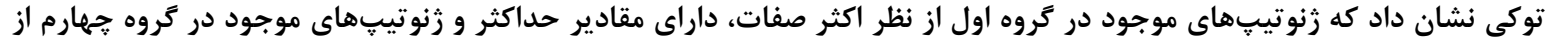

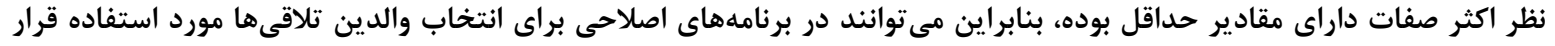

خوى در استان آذربايجان غربى با سطح زير كشتى معادل

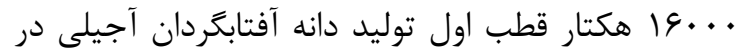

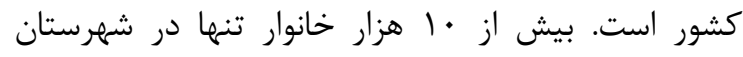

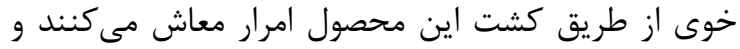

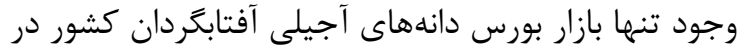

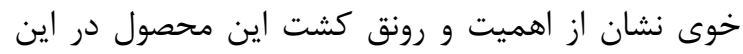

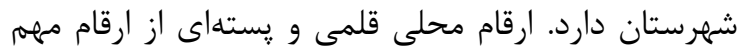

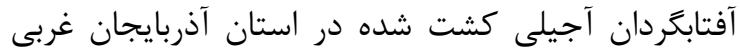

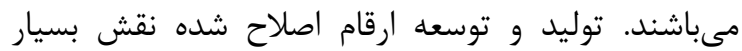

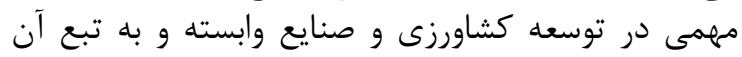
توسعه اقتصادى استان دارد.

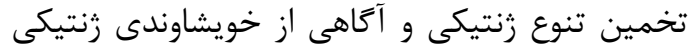

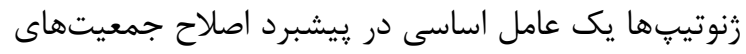

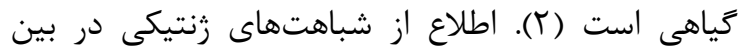

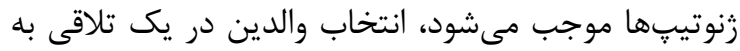

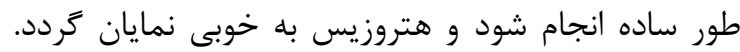

آفتابگردان زراعى (Helianthus annuus L.) متعلق به

جنس هليانتوس و خانواده Asteraceae يا

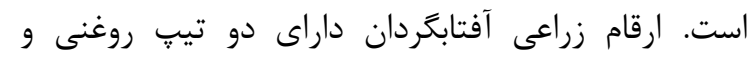

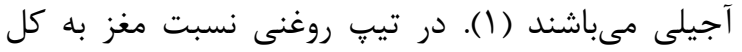

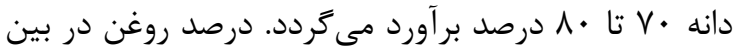

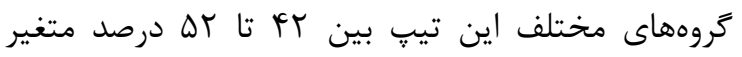
بوده و براى استحصال روغن مورد استفاده قرار ميى كيرند.

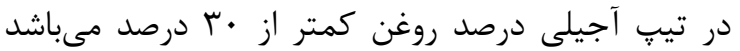

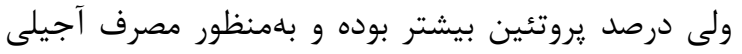

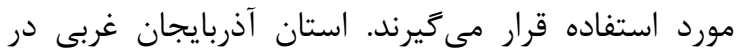

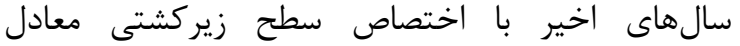

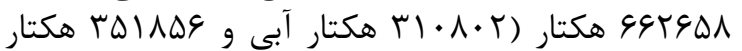

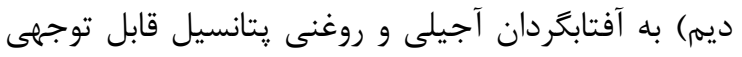

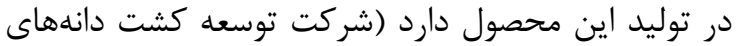

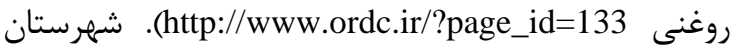


(Y) (Prunus persica L. Batsch) (Nicotiana (Yelianthus annuus L.) استفاده شدهاند. اين يزوهش بامنظور (YV) tabacum L.)

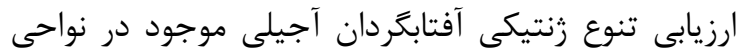

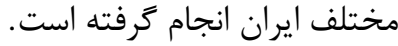

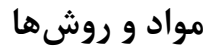 مواد كياهى و طرح آزمايشات}

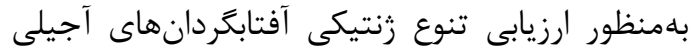

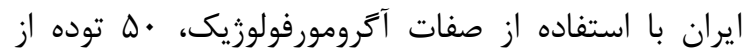

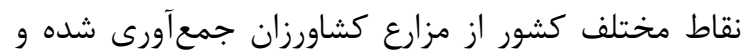

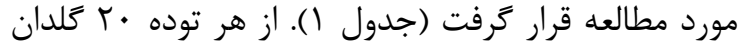

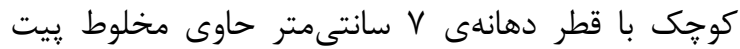

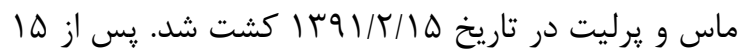

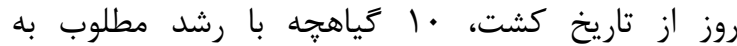

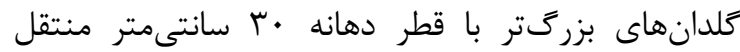

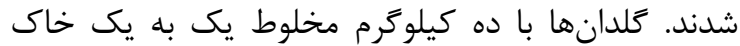

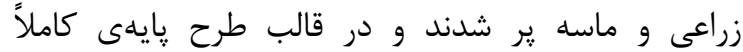

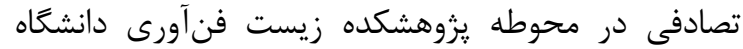

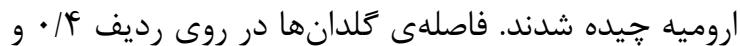

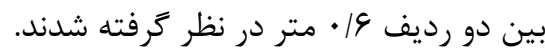

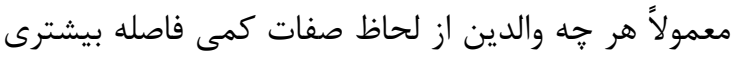

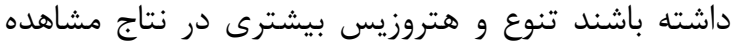

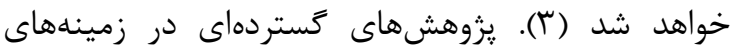

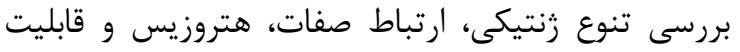

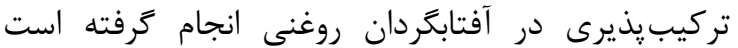

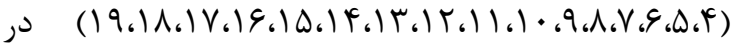

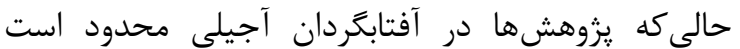
. ( $(\mathrm{r} \cdot)$

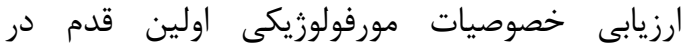

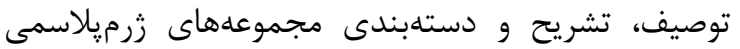

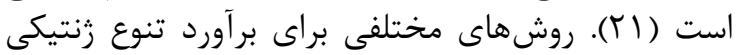

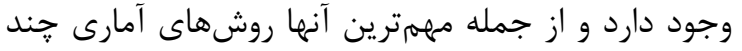

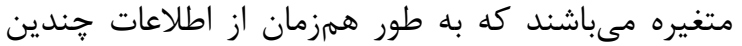

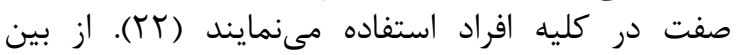

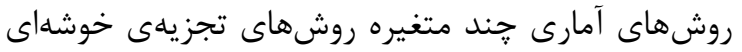

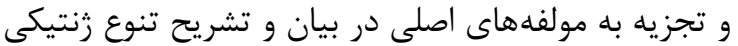

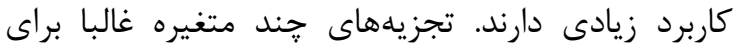

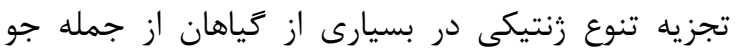

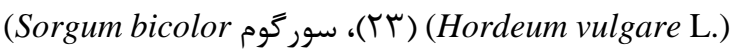
(Y) (Triticum spp) (YY) L. Moench)

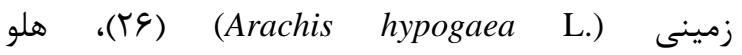

\begin{tabular}{|c|c|c|c|c|c|c|c|}
\hline عرض جغرافيايى & طول جغرافيايى & محل جمع آورى & كدتوده & عرض جغرافيايى & طول جغرافيايى & محل جمعآورى & كل كد توده \\
\hline$r 4^{\circ} / Q^{\prime}$ & Fér. & سقز 1 & TE & $r v^{\circ} f^{\prime}$ & $40^{\circ} \cdot$ & اروميه (انگنه \&) & 11 \\
\hline$r 4^{\circ} 10^{\prime}$ & rq.T. & سقز r & $\wedge$ & $r v^{\circ} Y \cdot{ }^{\prime}$ & $40^{\circ} \cdot$ & اروميه (اردوشاهى () & $\Delta$ \\
\hline$r \varphi^{\circ} 1 Q^{\prime}$ & HE.T. & سقز r & fr & $r V^{\circ} F^{\prime}$ & $F \Delta^{\circ} \cdot$ & اروميه (باباكنجه 9) & fF \\
\hline$r \varphi^{\circ} 10^{\prime}$ & 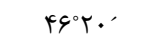 & سقز & it & $r v^{\circ} Y .^{\prime}$ & $F \Delta^{\circ} \cdot$ & اروميه (باروج) & $\varphi_{\Delta}$ \\
\hline$r \varphi^{\circ} 1 \omega^{\prime}$ & HQT. & سقز هـ & (i) & $r v^{\circ} F^{\prime}$ & $40^{\circ} \cdot$ & اروميه (جبل كندى r) & r) \\
\hline «ペリ' & fFop & سلماس (صدقيان) & 10 & $r v^{\circ} \mathcal{F} .^{\prime}$ & $F 0^{\circ} \cdot$ & اروميه (علىبحلو) & is \\
\hline r片l' & fropt & سلماس (قرهقلاق- بادامى) & 1. & $r v^{\circ} Y \cdot{ }^{\prime}$ & $r \Delta^{\circ} .^{\prime}$ & اروميه (للو ه) & $\Delta \cdot$ \\
\hline "ペリ' & fFop & سلماس (قرهقشلاق_قلمى) & 4 & rG०H & $F D^{\circ} \mid Y^{\prime}$ & اروميه (مرنخلو- منجخلو) & Fr \\
\hline$r \Lambda^{\circ} \mid V^{\prime}$ & $r Q^{\circ} Y G^{\prime}$ & سلماس (قزلجه r) & 9 & $r V^{\circ} \Delta \mu^{\prime}$ & $F D^{\circ} r q^{\prime}$ & اروميه (نوشينشهر) & r \\
\hline rם' & |l' & سندج & fq & $r v^{\circ} Y \cdot{ }^{\prime}$ & $F \Delta^{\circ} \cdot$ & اروميه (وقاصلوىسفلى) & rq \\
\hline rG'rd' & $\Delta F^{\circ} \Delta \Lambda^{\prime}$ & شاهرود 1 & rq & $r v^{\circ} \mathcal{H}^{\prime}$ & $\forall Q^{\circ} \cdot$ & اروميه (وقاصلوى اوليا) & $r$. \\
\hline rG'T & $\Delta F^{\circ} \Delta \Lambda^{\prime}$ & شاهرود r & rv & ים & DI & اصفهان (دولتآباد) & IV \\
\hline $\mathrm{M \Lambda}^{\circ}$ & $r \Delta^{\circ}$ & شبستر (كوزهكنان () & r & 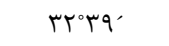 & DI ${ }^{\circ} \mathrm{F}{ }^{\prime}$ & اصفهان (طالخونجه) & if \\
\hline$r \Lambda^{\circ}$ & $4 \Delta^{\circ}$ & شبستر (كوزهكنان r) & rt & $r \Delta^{\circ} \Delta q^{\prime}$ & $F Q^{\circ} \Delta H^{\prime}$ & بانه 1 & rI \\
\hline$r \Lambda^{\circ}$ & $r \Delta^{\circ}$ & شبستر (كوزهكنان r) & ra & $r \Delta^{\circ} \Delta q^{\prime}$ & $F \Delta^{\circ} \Delta H^{\prime}$ & بانه r & זr \\
\hline " & $\Delta r^{\circ} \cdot$ & مازندران (تيرتاش) & re & $r \Delta^{\circ} \Delta q^{\prime}$ & $F \Delta^{\circ} \Delta H^{\prime}$ & بانه r & rA \\
\hline ґАץ.' & $F \Delta^{\circ} \mathrm{FQ}{ }^{\prime}$ & مرند |- 9یرץ| & f. & 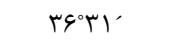 & $4 \varphi^{\circ} \mid r^{\prime}$ & بوكان & r \\
\hline rペr.' & $F D^{\circ} Y Q^{\prime}$ & مرند ז- 91ץ| & rt & 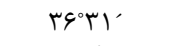 & $\left.r \varphi^{\circ}\right|^{\prime}$ & بوكان محلى & f \\
\hline ґ^ґ.' & $F Q^{\circ} \mathrm{FQ}$ & مرند (ديزج- قلمى) & re & $r \varphi^{\circ}$ & $\varphi \Delta^{\circ}$ & ييرانشهر (انديزه) & $\checkmark$ \\
\hline$r \Lambda^{\circ} \varphi^{\prime}$ & $Y Q^{\circ} \mathrm{FQ}$ & مرند (يامجى- קֶتهاى) & tr & $r 4^{\circ}$ & $\varphi \Delta^{\circ}$ & ييرانشهر (بالابان) & 1 \\
\hline rद.r.' & $\Delta q^{\circ} T \Delta^{\prime}$ & مشهد & r & $r \varphi^{\circ}$ & $4 a^{\circ}$ & ييرانشهر (سروكانى) & 19 \\
\hline$M F^{\circ} 19^{\prime}$ & $Y \Lambda^{\circ} \Delta I^{\prime}$ & ملاير سفيد r & ry & $r \Lambda^{\circ} \boldsymbol{L}^{\prime}$ & $r \Delta^{\circ} \ldots$ & خوى 4 & Fr \\
\hline$r v^{\circ} r$. & FV ${ }^{\circ} \boldsymbol{F}^{\prime}$ & ميانه (بسين) & 19 & $r \Lambda^{\circ} r \omega^{\prime}$ & $r \Delta^{\circ}{ }^{\circ} \cdot$ & خوى ·1 & TD \\
\hline$r Y^{\circ} \Delta Y^{\prime}$ & Fヘำ' & همدان 1 & TF & אדיז' & $Y \wedge^{\circ} \Delta Y^{\prime}$ & سردشت & ऍ \\
\hline MYOQL & FNOT' & همدان r & $r$. & $r 4^{\circ} 10^{\prime}$ & Fq. & سقز & $\forall \wedge$ \\
\hline
\end{tabular}




\section{نتايج و بحث}

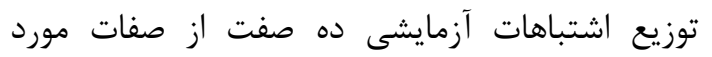

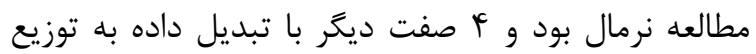

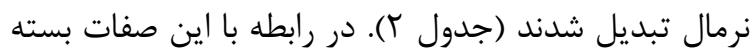

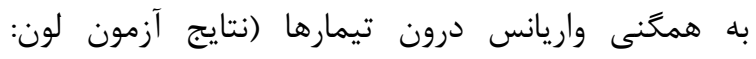
(Levene test

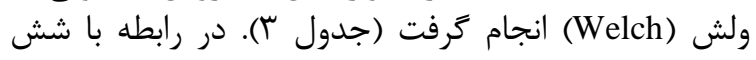

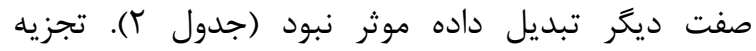

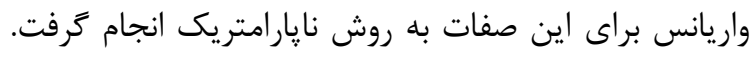

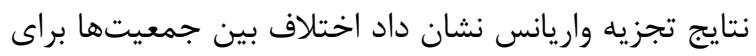

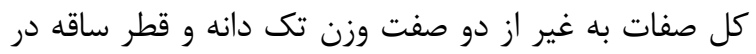

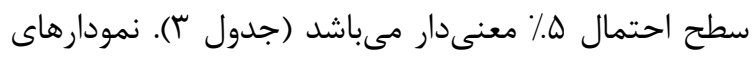

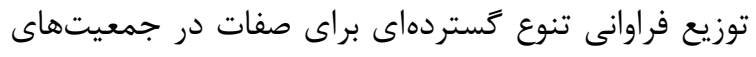

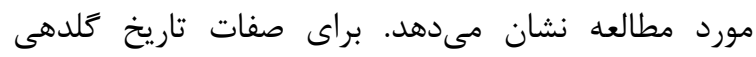

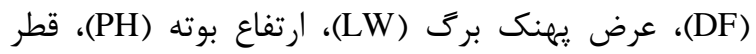

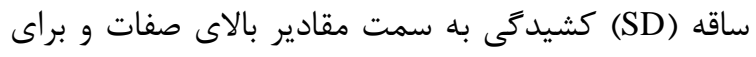

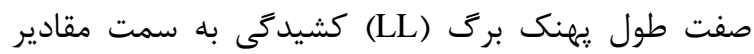

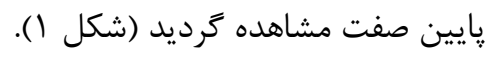

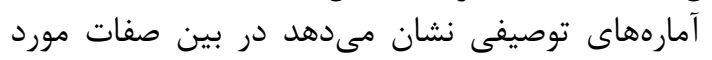

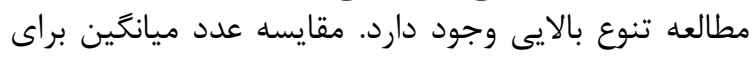

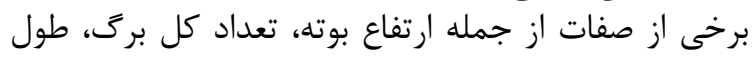

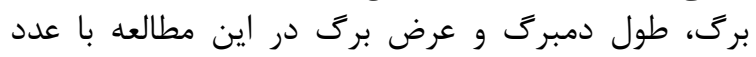

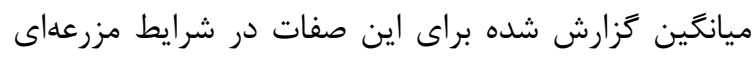

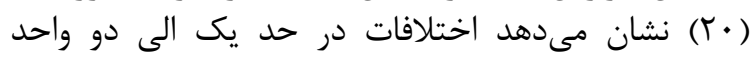

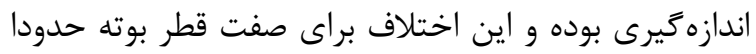

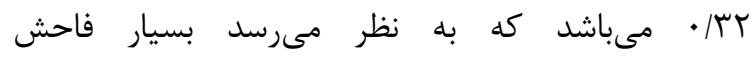

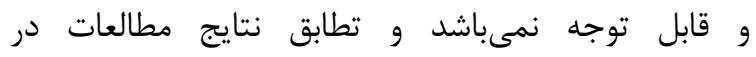

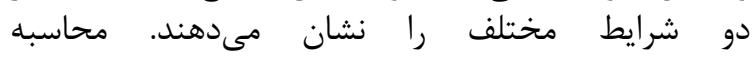

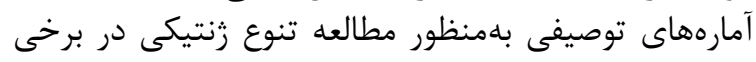
از محصولات ديخر از جمله سير (Allium sativum L.)

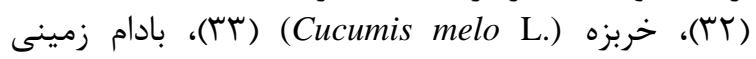
بامبارا (Vigna subterranea (L.) (Bambara groundnut)

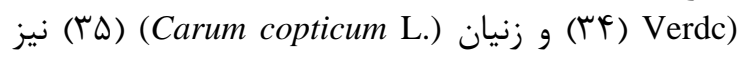

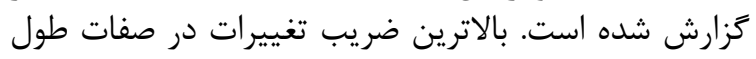

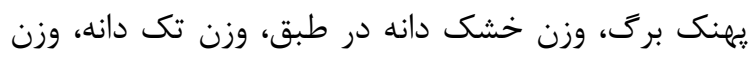

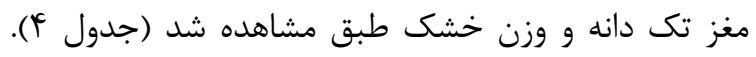

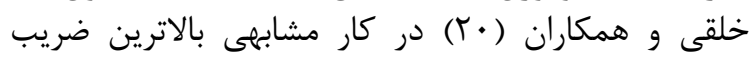

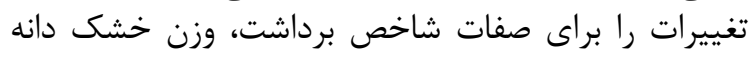

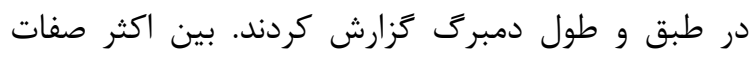

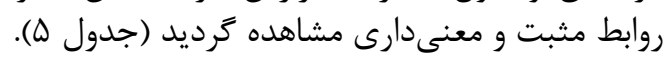

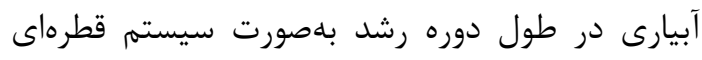

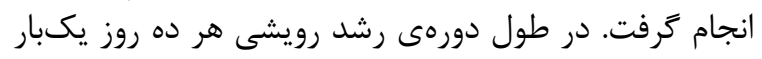

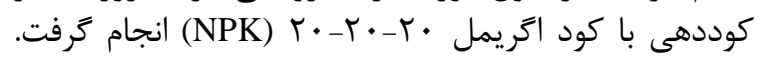

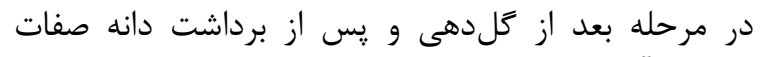

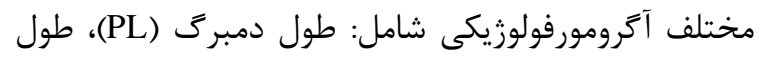

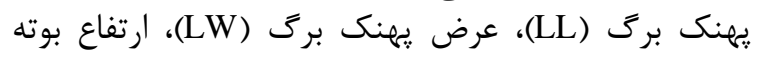

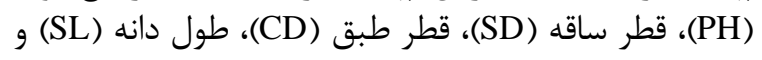

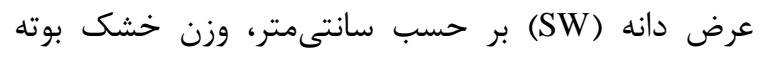

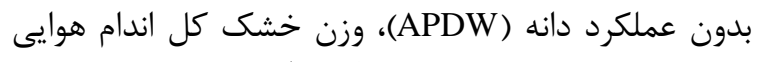

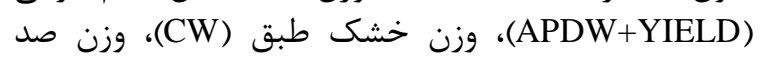

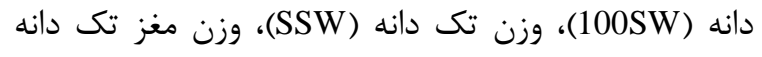
و وزن خشك دانه در طبق (DHW)

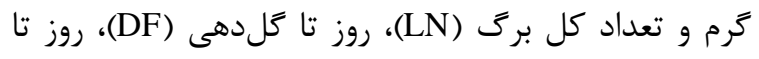

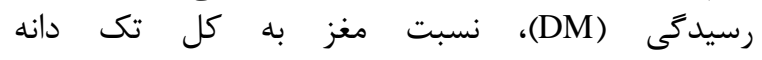

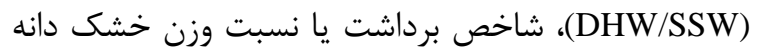

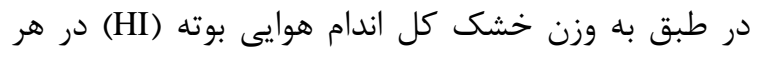

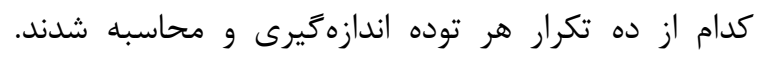

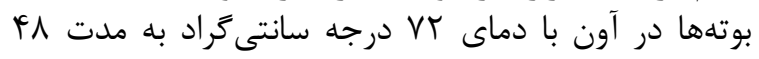

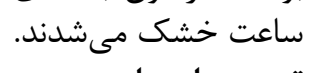

تجزيه دادهها

شناسايى داده(هاى) يرت (Outliers) و و آزمون نرمال

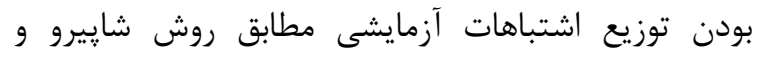

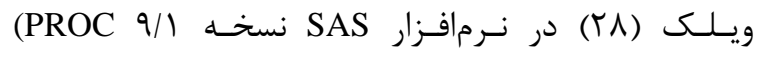

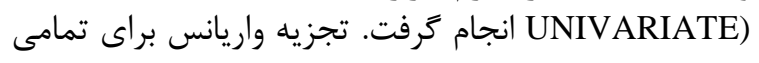

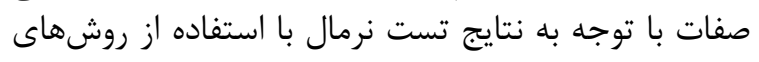

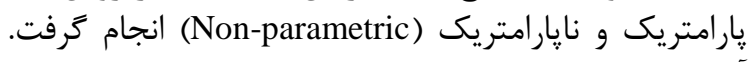

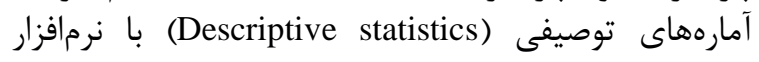
SPSS 20

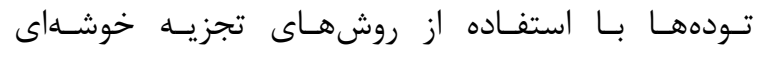

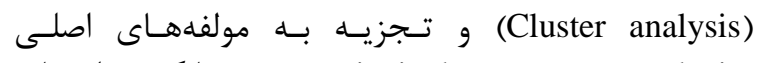
(Principal component analysis) اصلى انجام كرفت. تجزيه خوشهاى، يس از استاندارد كردن ماند

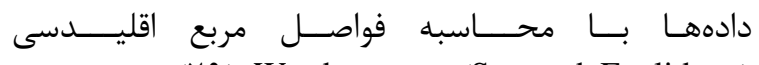
(Squared Euclidean)

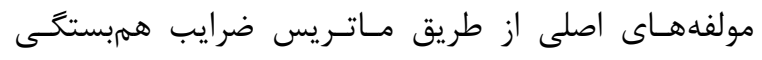

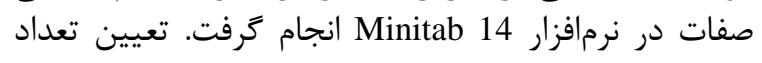

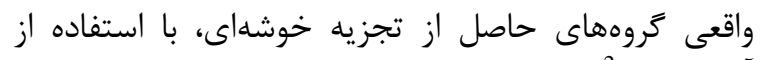

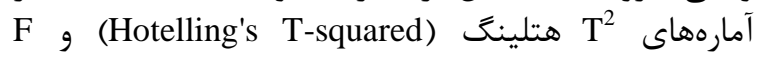

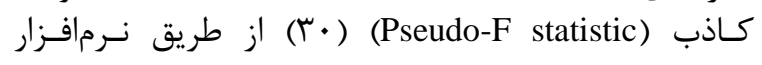

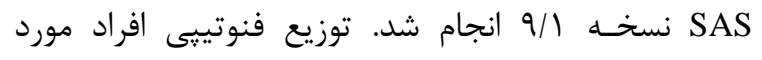

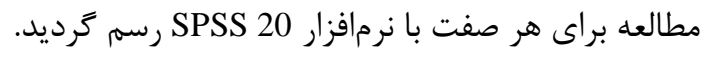


جدول r- آزمون توزيع نرمال اشتباهات آزمايشى و همخنى واريانس درون تيمارها بهمنظور ارائه روش تجزيه واريانس مناسب براى

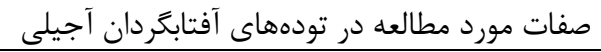

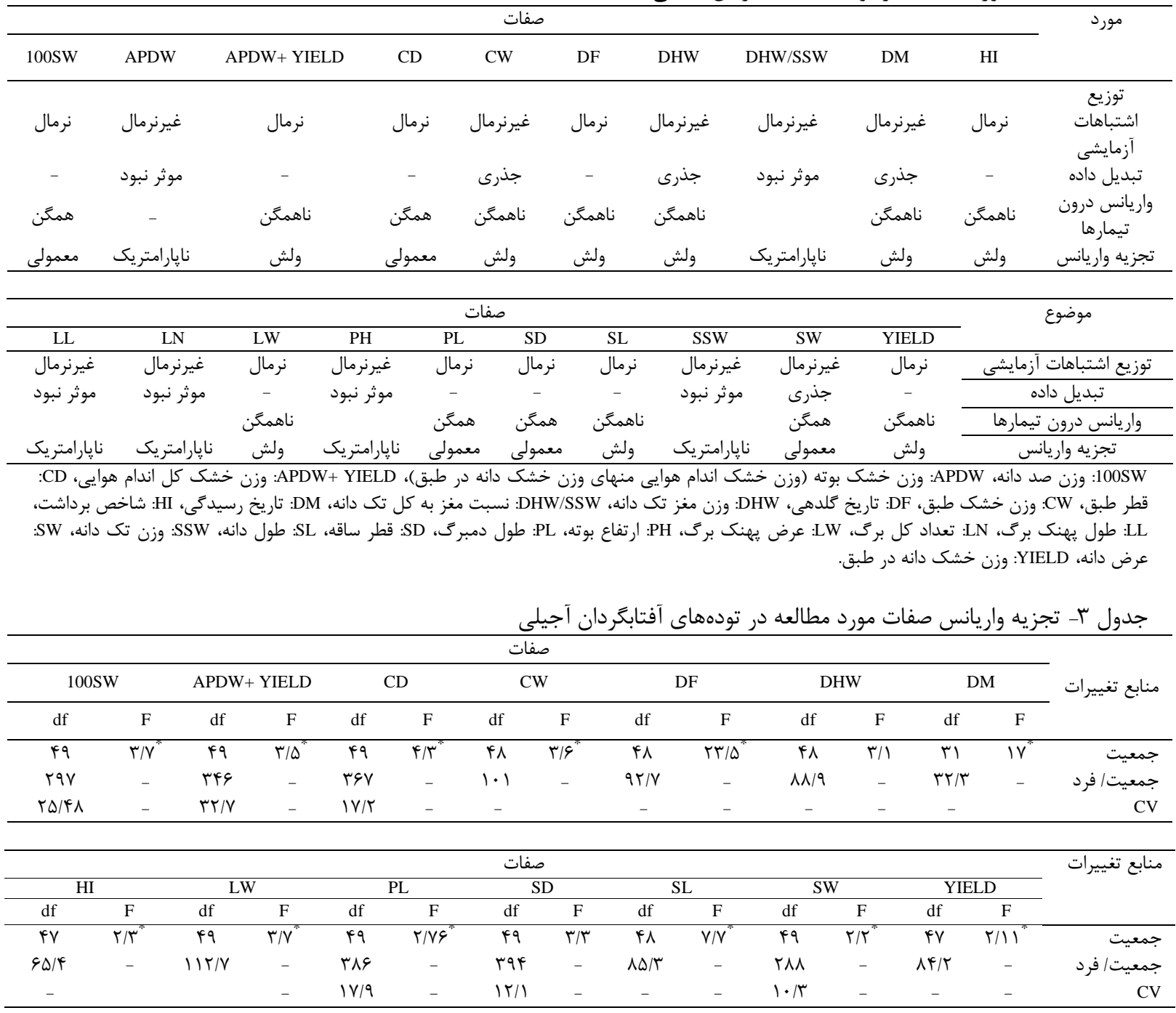

\begin{tabular}{|c|c|c|c|c|c|c|c|c|c|c|c|c|}
\hline \multicolumn{12}{|c|}{ صفات } & \multirow{3}{*}{ منابع تغييرات } \\
\hline \multicolumn{2}{|c|}{ APDW } & \multicolumn{2}{|c|}{ DHW/SSW } & \multicolumn{2}{|c|}{ LL } & \multicolumn{2}{|c|}{ LN } & \multicolumn{2}{|c|}{$\mathrm{PH}$} & \multicolumn{2}{|c|}{ SSW } & \\
\hline df & $\mathrm{F}$ & df & $\mathrm{F}$ & df & $\mathrm{F}$ & df & $\mathrm{F}$ & df & $\mathrm{F}$ & df & $\mathrm{F}$ & \\
\hline is & $r r / I^{*}$ & $4 q$ & $r / 1^{*}$ & $4 q$ & $11 / 4^{\prime \prime}$ & $4 q$ & $r T / I^{*}$ & is & $r \varepsilon / 9^{\circ}$ & $4 q$ & $r F / V^{\pi}$ & جمعيت \\
\hline THE & - & rar & - & rAs & - & rq. & - & rvi & - & rar & - & جمعيت/ فرد \\
\hline- & & - & & - & & - & & - & & - & & $\mathrm{CV}$ \\
\hline
\end{tabular}

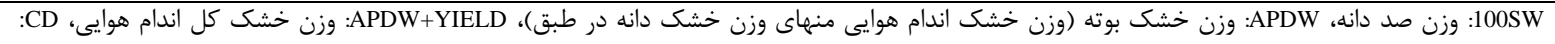

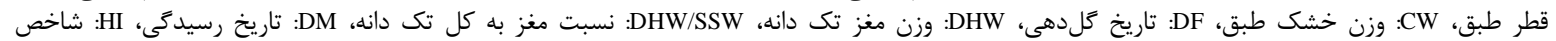

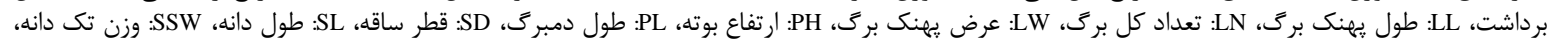

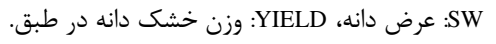




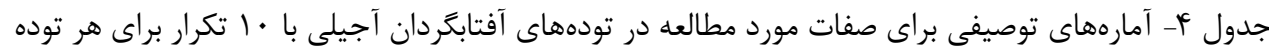

\begin{tabular}{|c|c|c|c|c|c|}
\hline ميانكَين & انحراف معيار & حداقل & حداكثر & ضريب تغييرات & صفات \\
\hline 9/AV & $1 / A \Lambda$ & $\Delta / 9 T$ & $14 / 91$ & $T Q / F A$ & $100 \mathrm{SW}(\mathrm{gr})$ \\
\hline$|r v /| f^{f}$ & Tr/QT & $9 \pi / 94$ & $194 / 11$ & TY/AD & APDW (gr) \\
\hline $19 / 9$ & T/K & $11 / 9$. & $r / / r$. & $I V / r$. & CD (cm) \\
\hline$r / / v$ & $9 / 41$ & $\mid N / \cdot T$ & $F F / r \Lambda$ & $r \Delta / \mu^{\kappa}$. & $\mathrm{CW}(\mathrm{gr})$ \\
\hline$N V / F q$ & GIVT & $9 V / r$. & $\cdot|4|$ & GNV & DF \\
\hline$\cdot \pi \Delta$ & .1 .9 & $\cdot / l F$ &.$|4|$ & $r 9 / 91$ & DHW (gr) \\
\hline$\cdot 109$ & .1 .4 & $\cdot / F V$ &. $\mid 9 \mathrm{~V}$ & IN/KF & DHW/SSW \\
\hline$\cdot / r \Lambda$ & .1 .4 & $\cdot / / V$ & .44 & $V / 99$ & HI \\
\hline$r r / \cdot \Lambda$ & $0 / 19$ & $19 / .$. & $\Delta V / \cdot$. & rT/FA & $\mathrm{LL}(\mathrm{cm})$ \\
\hline rI/qT & $r / 91$ & $r T / l$. & $F r / .$. & $F T / L Q$ & LN \\
\hline$r \cdot \pi r$ & $1 / 4 v$ & $19 / 0$ & $r T / V$ & $|F| \Lambda \mid$ & LW (cm) \\
\hline $194 / \Delta T$ & $r \mid / r V$ & $1 \pi q / r$ & TrT/Y & $11 / \pi r$ & $\mathrm{PH}(\mathrm{cm})$ \\
\hline $\mid F / . F$ & $1 / 4$. & $1 \cdot / 19$ & $19 / \Lambda \%$ & $|V / 9|$ & $\mathrm{PL}(\mathrm{cm})$ \\
\hline$V / \Delta \Lambda$ & $\cdot 109$ & $\Delta / 9$. & $1 / r^{\prime} q$ & $\mid r / I r$ & $\mathrm{SD}(\mathrm{cm})$ \\
\hline $\mid N / 1$. & $T / T V$ & $\mid \varphi / \cdot$. & $r F /$. & $\mid F / T F$ & $\mathrm{SL}(\mathrm{cm})$ \\
\hline$\cdot / F F$ & $\cdot 111$ & $\cdot|r|$ & $\cdot(N)$ & rV/a9 & SSW (gr) \\
\hline$V / 99$ & $\cdot 119$ & $91 .$. & $9 / 94$ & $r \cdot / \Delta F$ & $\mathrm{SW}(\mathrm{gr})$ \\
\hline$\Delta T / T F$ & $|r / \pi|$ & זq/१ & $V Y / \Delta \Delta$ & $F \cdot / V q$ & YIELD (gr) \\
\hline
\end{tabular}

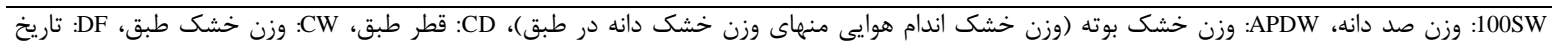

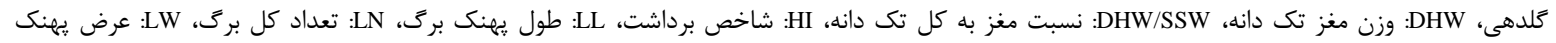

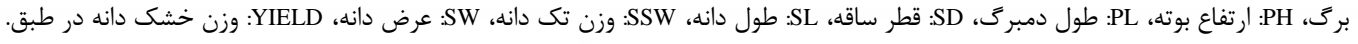
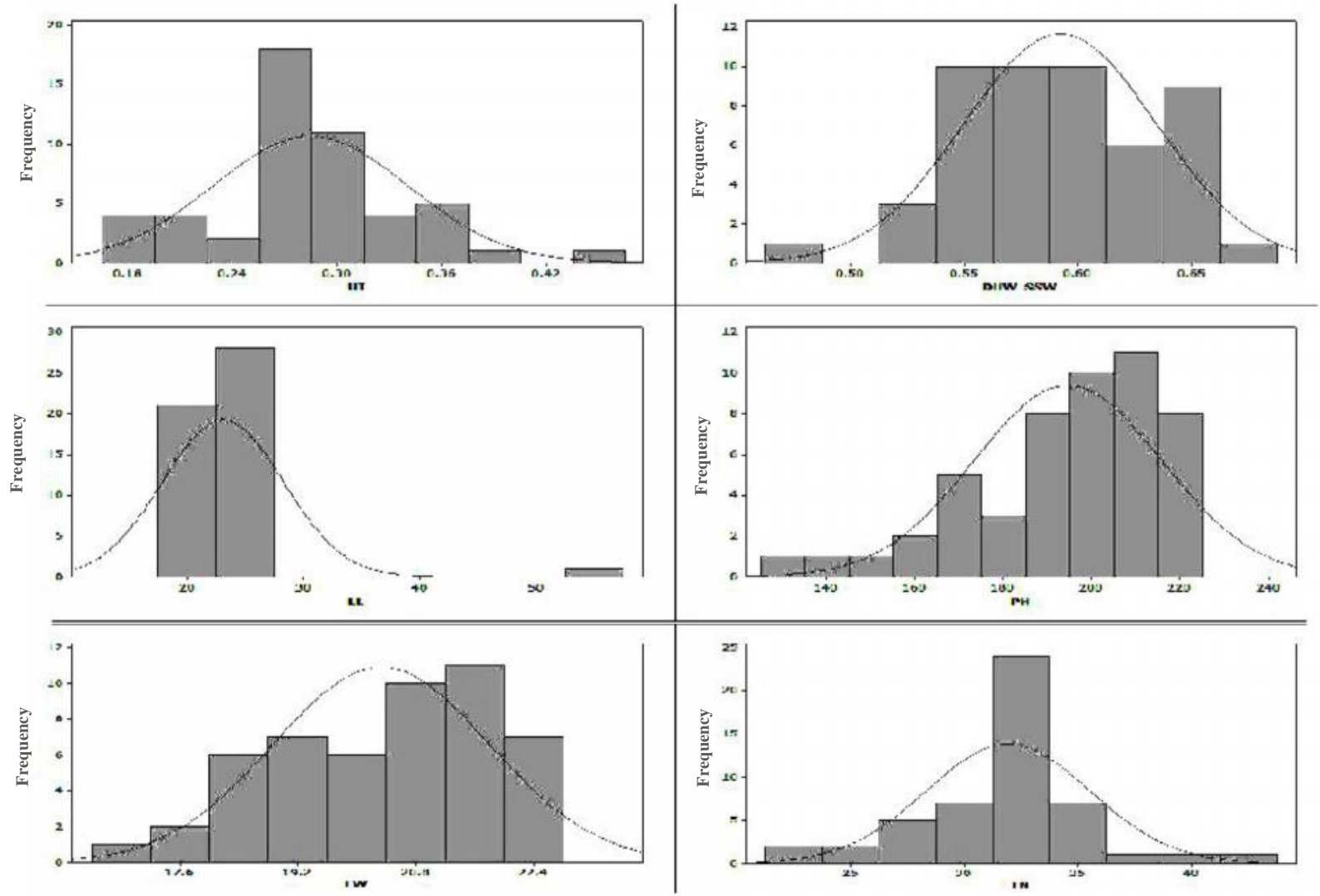

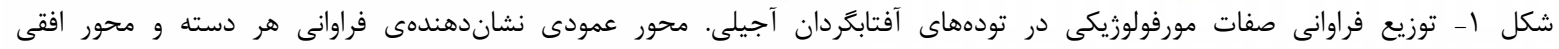

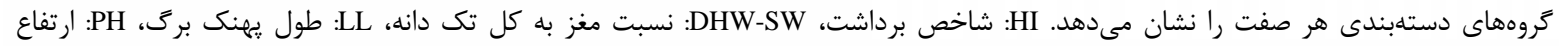

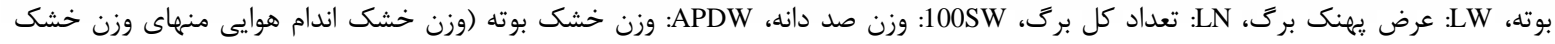

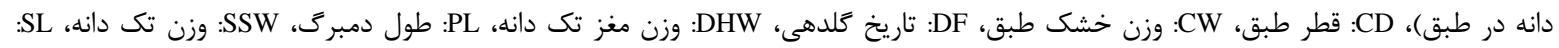

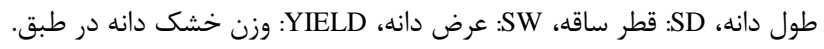



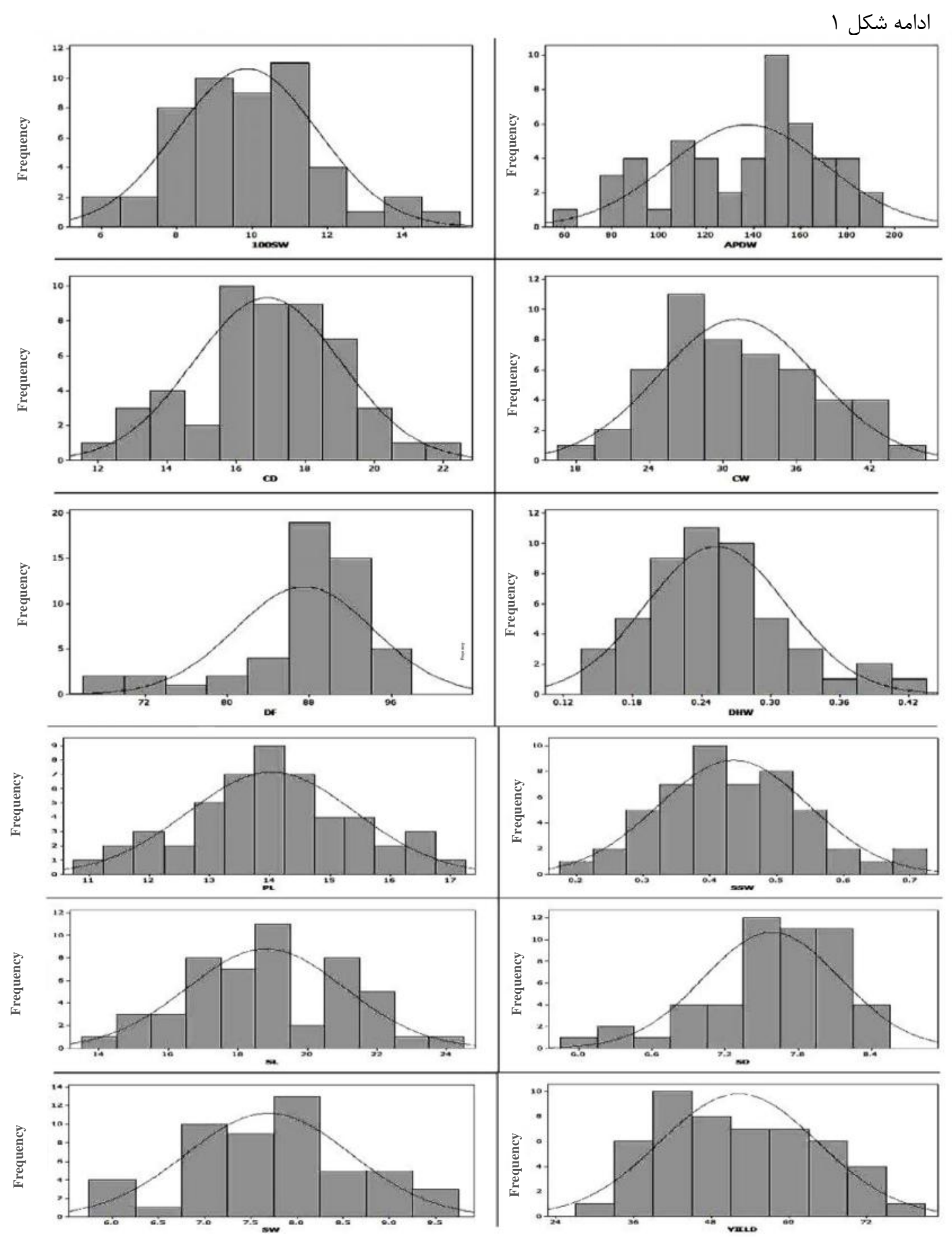
iᄉ بررسى تنوع زنتيكى تودههاى آفتابكردان آجيلى بر اساس صفات آكرومورفولوزيك با استفاده از روشهاى آمارى خند متغيره ....

جدول ه- همبستخى بين صفات مورد مطالعه در تودههاى آفتابخردان آجيلى

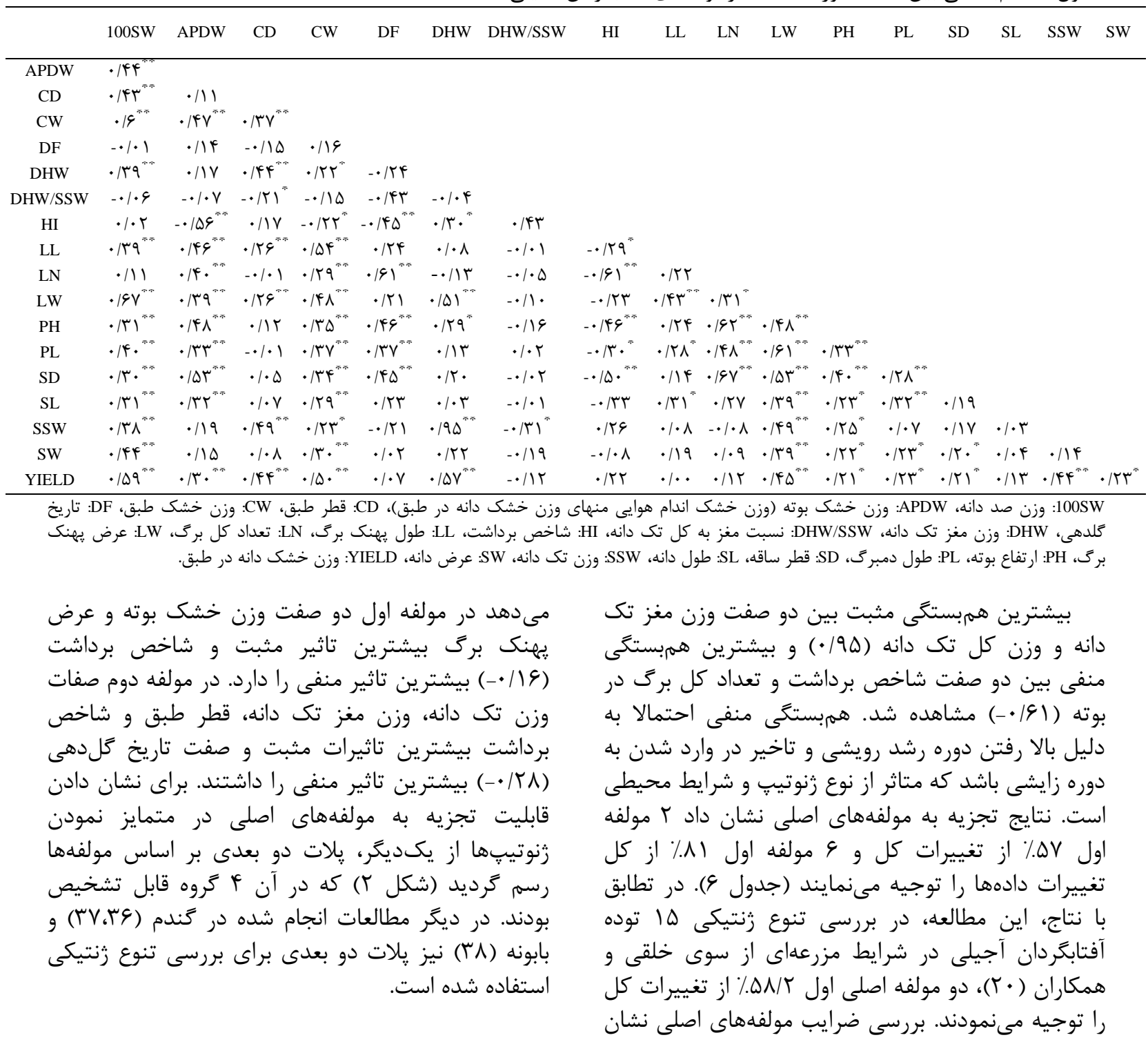


جدول צ- تجزيه به مولفههاى اصلى روى صفات آترومورفولوزيكى در تودههاى آفتابگردان آجيلى

\begin{tabular}{|c|c|c|c|c|c|c|c|c|}
\hline \multirow[t]{2}{*}{ متغير } & \multicolumn{8}{|c|}{ مولفه } \\
\hline & اول & دوم & سوم & جهارم & ينجم & ششم & هفتم & هشتم \\
\hline YIELD & $\cdot / F^{F}$ & $\cdot / T \Delta$ & $.1 \cdot 9$ & $\cdot \cdot / / V$ &.$/ \pi$ & - $\cdot / K T$ & $\cdot 1 \cdot \Delta$ & $-\cdot / 1$ \\
\hline $100 \mathrm{SW}$ & $\cdot / r \Lambda$ & $\cdot / r$ & $-\cdot / T r$ & $\cdot / 1 \Delta$ & -.119 & -.119 & .119 & $.1 \cdot V \Delta$ \\
\hline APDW & (Tr & -.111 & .11 & . & -.111 & $-.1 \cdot 9$ &.$- / r \wedge$ & $-\cdot / T V$ \\
\hline $\mathrm{CW}$ & $\cdot|\mu|$ & $\cdot 1 \cdot V$ & $-.1 \cdot v$ & $\cdot / \cdot F$ & $\cdot|\pi|$ &.$/ \mu F$ & -.1 .1 & $\cdot \cdot / r V$ \\
\hline $\mathrm{HI}$ & -.119 & $\cdot \pi V$ & $-\cdot 1 \cdot \Delta$ & $-\cdot 110$ & $\cdot|r|$ & $-.1 \cdot 9$ & $\cdot / K F$ & $\cdot / \mu F$ \\
\hline $\mathrm{CD}$ & $\cdot / 11$ & 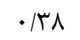 &. .1 .9 & $.1 . r q$ & $\cdot / 149$ & $\cdot / 1$ &.$- / 1$ & 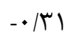 \\
\hline $\mathrm{LN}$ & $\cdot \mid / 4$ & $\cdot \cdot / \pi \varphi$ & r &.$/ 1 T$ & $\cdot / 1 \Delta$ & $\cdot / 14$ & .1 .9 &.$/ 14$ \\
\hline LL & .119 & -.1 .9 & $-\cdot / 4 \wedge$ & $\cdot / 49$ & $-\cdot / 11$ & $\cdot|4|$ & $\cdot / \mu \wedge$ & $\cdot \cdot / r \Lambda$ \\
\hline LW & (Tr & .1 .9 & $-\cdot / 1 \mathrm{~V}$ &. .1 .4 &.$- / \cdot f$ & $\cdot / 1 Q$ & $\cdot 1 \cdot r$ & $\cdot / \cdot \Delta$ \\
\hline PL & $\cdot / r \Lambda$ & $\cdot \cdot / f$ &.$/ 1 f$ & $-\cdot 1 \cdot v$ & $\cdot / l V$ & -.1 .9 & .110 & $\cdot 109$ \\
\hline SD & $\cdot / r \Lambda$ & $. \cdot / 1 T$ & rr/. & $-\cdot / r q$ &.$- / 49$ & $-\cdot / \cdot r$ &.$- / 1$ &.- .1 .1 \\
\hline PH & • & $-\cdot 1 \cdot 1$ & $\cdot|r|$ & $-\cdot \cdot 1 \cdot 1$ & $-\cdot / \cdot V$ & $\cdot / T F$ & $-\cdot / \cdot V$ & $\cdot / 1 \wedge$ \\
\hline DF & $\cdot 110$ & $-\cdot / T \Lambda$ & $.1 \cdot \mathrm{f}^{2}$ & $-\cdot 1 \cdot V$ & $\cdot / F F$ & .111 & $\cdot \pi v$ & r//r \\
\hline $\mathrm{DM}$ & $\cdot / r \Delta$ & Tr/. & $\cdot / 11$ & $\cdot / r V$ & $\cdot / 1 r$ & $\cdot / \cdot 4$ & $\cdot 1 \cdot 1$ & $\cdot / \pi 9$ \\
\hline SSW & .119 & . & $\cdot / 1 F$ & $. \cdot . \cdot r$ &. .111 & 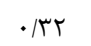 & -.1 .9 & $\cdot / \cdot V$ \\
\hline DHW &.$/ 18$ & . & $\cdot / \cdot \Delta$ & $\cdot \cdot \pi r$ &.$- / 1 V$ & . & $\cdot / \cdot r$ & $\cdot / \cdot \Lambda$ \\
\hline DHW/SSW & $-.1 \cdot \Delta$ & $. \cdot / 1 T$ & $-\cdot / 4 q$ & -.199 &.$- / T V$ &.$- / 4$ & سאן. &.$- / 1$ \\
\hline SL &.$/ 19$ &.$\cdot 111$ & $\cdot \cdot|\Delta|$ & $\cdot / \cdot r$ & $\cdot / \cdot r$ & $-.1 \cdot 9$ & $-\cdot / Q V$ & .119 \\
\hline SW & $\cdot / r$ & .111 & $\cdot \pi$ & . & - & $-\cdot / f F$ & $\cdot / T F$ & $. \cdot / \cdot r$ \\
\hline $\begin{array}{c}\text { مقادير ويزه } \\
\text { (Eigen values) }\end{array}$ & $V / \cdot 1$ & r/AV & $1 / \pi$ & $1 / T \Lambda$ & $1 / .9$ & $\cdot 119$ & . IVT & .109 \\
\hline واريانس نسبى & $\cdot / r v$ & $\cdot / r$ & $\cdot / \cdot V$ & $\cdot / \cdot V$ & .1 .9 & $\cdot 1 \cdot \Delta$ & $\cdot / \cdot f$ & . \\
\hline واريانس تجمعى & $\cdot / r V$ & $\cdot \mid \Delta V$ & $\cdot 194$ & $\cdot|V|$ & $\cdot / \mathrm{VV}$ & $\cdot|\lambda|$ & $\cdot / \wedge \Delta$ & .119 \\
\hline
\end{tabular}

طIELD

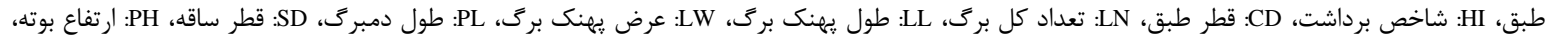

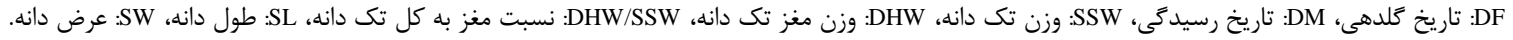

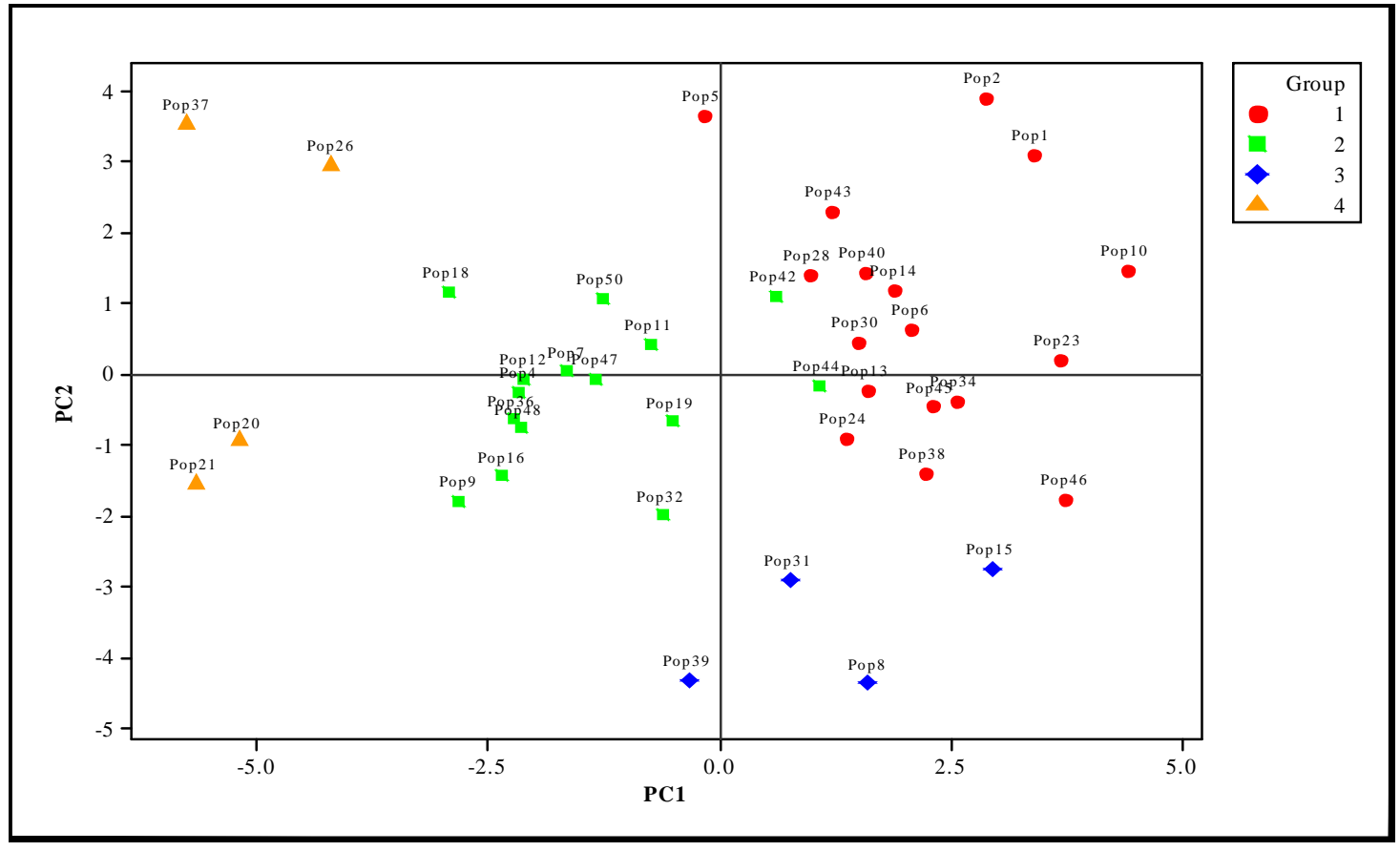

شكل r- دستهبندى تودههاى آفتابكردان آجيلى بر اساس دو مؤلفه اول و دوم تجزيه به مؤلفههاى اصلى روى صفات آكرومورفولوزيك. 
$\omega \cdot$ بررسى تنوع زنتيكى تودههاى آفتابكردان آجيلى بر اساس صفات آكرومورفولوزيك با استفاده از روشهاى آمارى جند متغيره ..

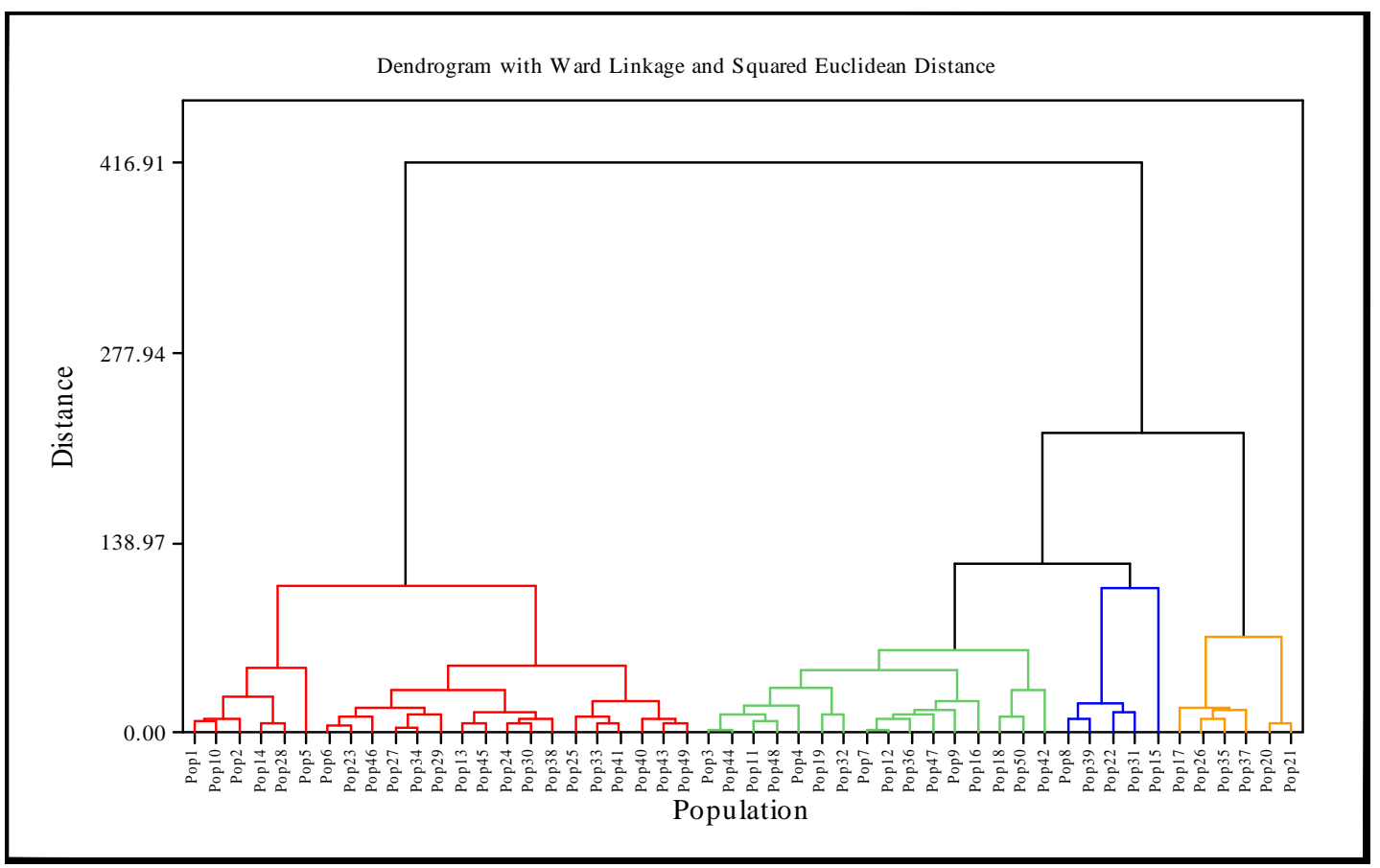

شكل سـ دندروگرام حاصل از تجزيه خوشهاى تودههاى آفتابگردان آجيلى بر اساس صفات آكرومورفولوزيك مورد مطالعه به روش وارد.

جدول V- مقادير T كاذب هتلينگ و Fاذب براى تعيين تعداد واقعى گروهها

\begin{tabular}{|c|c|c|c|c|}
\hline تعداد گروه & F كاذب F & كاذب هتلينگ T2 & Cluster X: Cluster Y & فاصله \\
\hline 4 & $9 / \Delta$ & $r / V$ & Cluster I: Cluster Y & $\mathrm{r} / \mathrm{QV}$ \\
\hline$\Delta$ & $\Lambda$ & v & Cluster I: Cluster $\Upsilon$ & $\varphi / \Lambda$. \\
\hline i & $1 \cdot / r$ & $r / \mu$ & Cluster I: Cluster ${ }^{\mathrm{f}}$ & $\mathrm{V} / \cdot$. \\
\hline r & $r / 9$ & $r \cdot / \Delta$ & Cluster $Y$ : Cluster $\Upsilon$ & $y / \cdot r$ \\
\hline r & $r / \Delta$ & $r / \Delta$ & Cluster Y: Cluster Y & 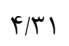 \\
\hline 1 & · & $r / \Delta$ & Cluster r: Cluster $₹$ & $V / 19$ \\
\hline
\end{tabular}

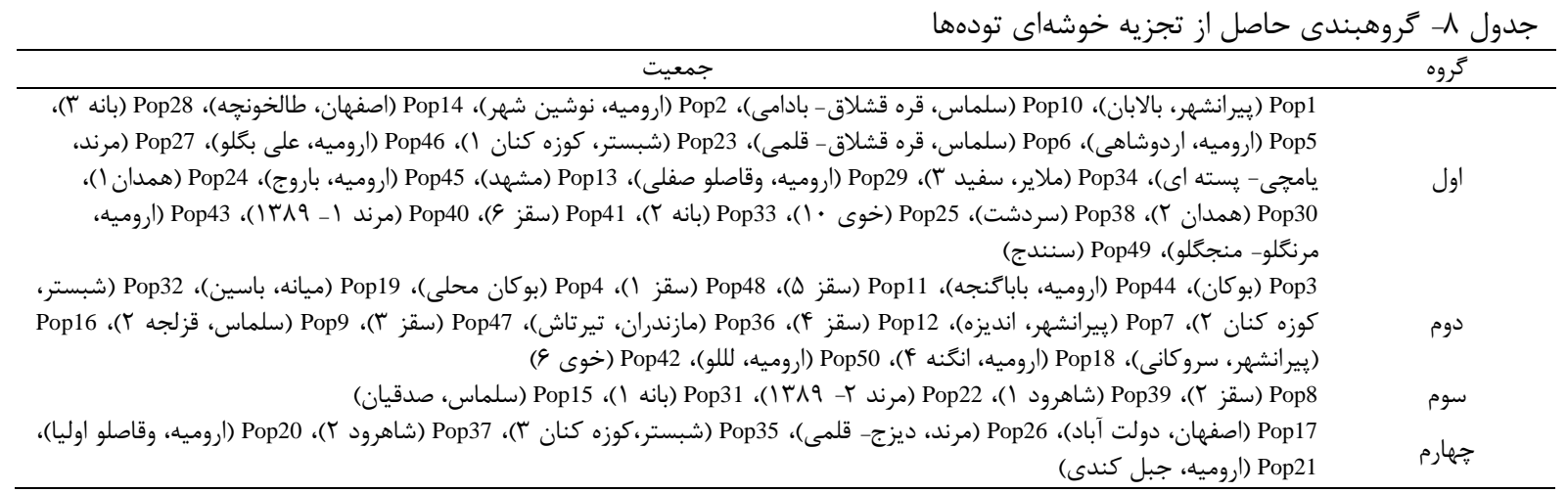




\begin{tabular}{|c|c|c|c|c|c|c|c|c|c|c|}
\hline LW & LL & $\mathrm{LN}$ & $\mathrm{CD}$ & $\mathrm{HI}$ & $\mathrm{CW}$ & APDW & 100SW & & تعداد زنوتيب & كروه \\
\hline$\Gamma I / r \Delta^{a}$ & $r r / \Delta \Lambda^{a b}$ & $r T / V \mu^{b}$ & $\mid 1 / \cdot 9^{\mathrm{a}}$ & $\cdot \pi \Lambda^{b}$ & $r \varphi / 19^{a}$ & $109 / \Gamma r^{a}$ & $1 \cdot / 91^{\circ}$ & & $r \mu$ & اول \\
\hline $19 / 9 r^{\mathrm{bc}}$ & $T M / \Delta \Lambda^{\mathrm{b}}$ & $\Gamma / / \Delta \varphi^{b}$ & $19 / \mathrm{V} \cdot{ }^{\mathrm{ab}}$ & $\cdot / \mu \cdot{ }^{b}$ & $r V / T^{b c}$ & $11 N / F V^{D}$ & q/. rab & & 19 & دوم \\
\hline$r \cdot / / \Delta^{\mathrm{ab}}$ & $r \Lambda / \Lambda \Lambda^{a}$ & $r v / r v^{a}$ & $\mid r / 99^{c}$ & $\cdot 119^{c}$ & $r q / \Lambda)^{b}$ & $|\Delta V /| r^{\mathrm{a}}$ & $q / / f^{a b}$ & & $\Delta$ & سوم \\
\hline $\mid N / F V^{c}$ & $r \cdot / r^{D}$ & $r Q / T T^{D}$ & $\mid \Delta / q^{\mathrm{DC}}$ & $\cdot / r \omega^{\mathrm{a}}$ & $r \mu / r K^{c}$ & $\Lambda \Delta / r^{\mathrm{c}}$ & $\Lambda / F V^{D}$ & & 4 & جهارم \\
\hline YIELD & SW & SL & DHW/SSW & DHW & SSW & DF & $\mathrm{PH}$ & SD & PL & كروه \\
\hline$G K / / \Lambda^{a}$ & $\Lambda / I r^{a}$ & $19 / 4 v^{a}$ & $\cdot \mid \Delta \Lambda^{D}$ & $\cdot / q^{a}$ & $\cdot|\Delta|^{a}$ & $\Lambda V / f \Delta^{a}$ & $r \cdot V|\Lambda|^{a}$ & $V / \Delta \Lambda^{a c}$ & $1 F / v q^{a}$ & اول \\
\hline$\varphi \Delta / \& \Delta^{\circ}$ & $V / r \Lambda^{a}$ & $\mid \Lambda / v^{a b}$ & $\cdot 1 \Delta \Lambda^{\mathrm{D}}$ & $\cdot / \pi r^{a b}$ & $\cdot / f \cdot a b$ & $\wedge 9 / \mathrm{V} \cdot{ }^{a}$ & $\mid N N / \Delta F^{D}$ & $V / F \Delta^{D}$ & $\mid r / \Delta \varphi^{a b}$ & دوم \\
\hline$r \Lambda / r r^{D}$ & $V / \Delta^{a}$ & $\mid \Lambda / V^{a b}$ & $\cdot \mid q^{\mu^{\mathrm{a}}}$ & $\cdot / T \cdot{ }^{D}$ & D & $9 r / 9 \Lambda^{\mathrm{a}}$ & $r \cdot r / \cdot \varphi^{a}$ & $\mathrm{~V} / \mathrm{q}^{\mathrm{a}}$ & $\mid r / \mu \Delta^{a}$ & سوم \\
\hline$r \psi / r q^{D}$ & $V / r q^{a}$ & $19 / 4 \Delta^{D}$ & $\cdot \mid q Y^{\mathrm{ab}}$ & $\cdot \pi r^{\mathrm{ab}}$ & $\cdot / \pi v^{D}$ & $V V / T I^{D}$ & $|\Delta| / \Delta \Delta^{c}$ & $\Leftrightarrow \mid \Delta \Lambda^{\mathrm{c}}$ & $\| T / 1 \theta^{D}$ & جهارم \\
\hline
\end{tabular}

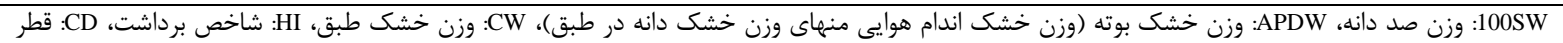

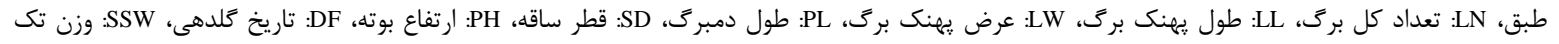

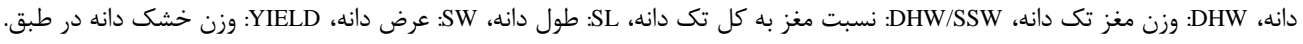

انتخاب والدين بر اساس تنوع زنتيكى مفيدتر مىباشد.

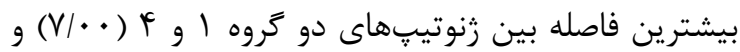

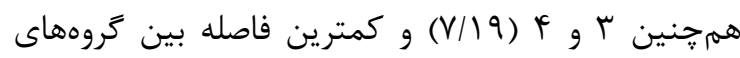

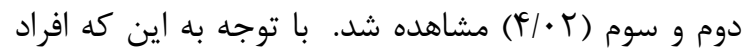

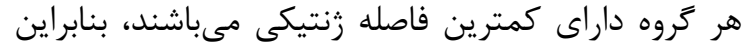

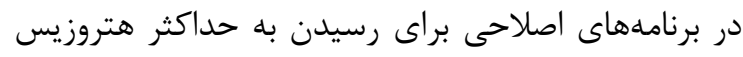

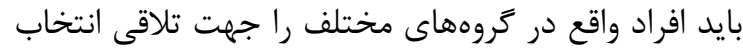

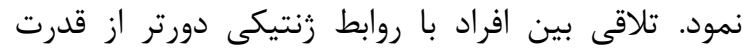

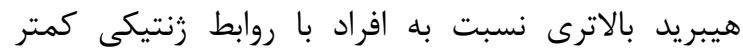

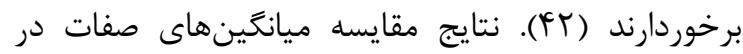

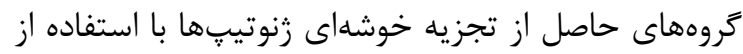

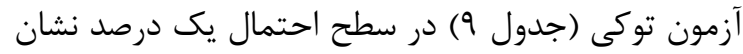

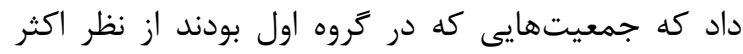

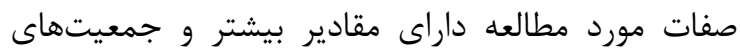

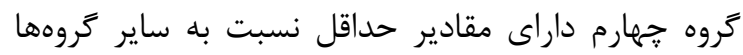

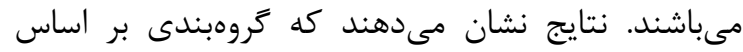

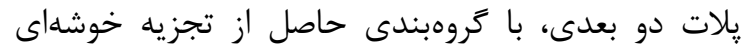

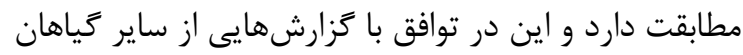

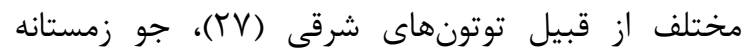

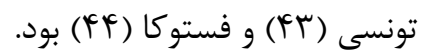

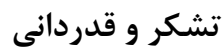

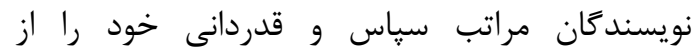

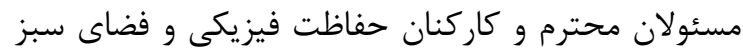

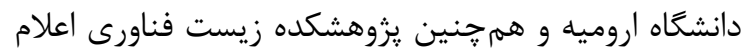

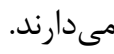

محققين براى انتخاب بهترين والدين در هر تلاقى در

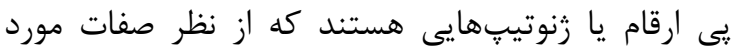

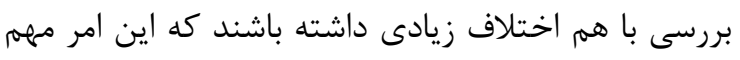

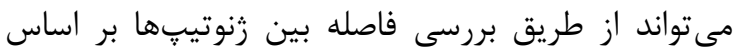

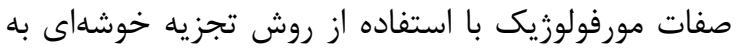

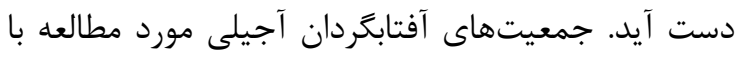

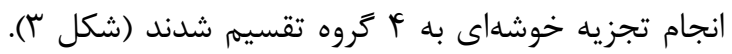

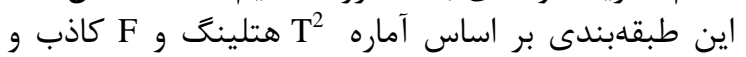

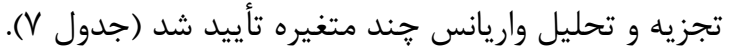
نتايج گروهبندى بهصورت واريانس واضح در جدول

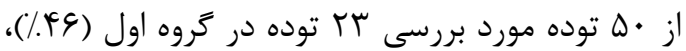

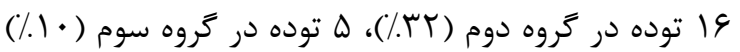

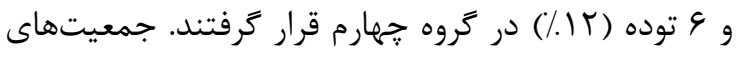

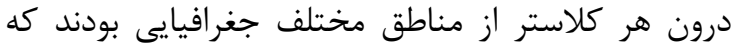

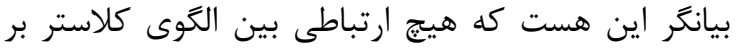

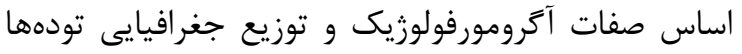

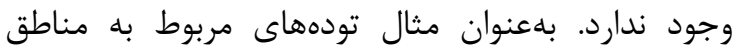

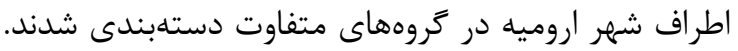

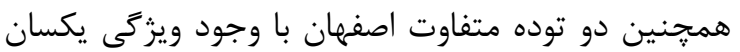

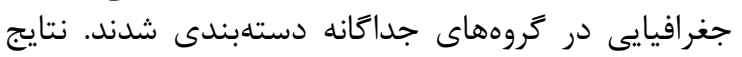

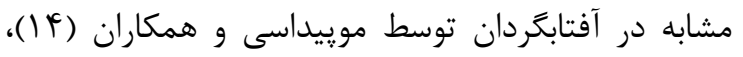

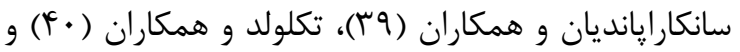

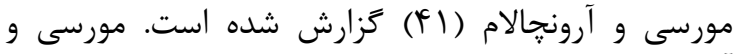

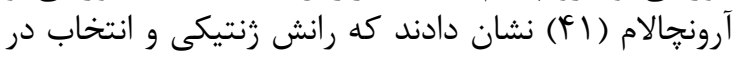

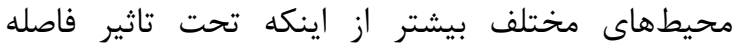

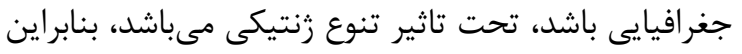


1. Hu, J., G. Seiler and C. Kole. 2010. Genetics, genomics and breeding of sunflower. Routledge, USA, 342 $\mathrm{pp}$.

2. Nikolic, D., V. Rakonjac, D. Milatovic and M. Fotiric. 2010. Multivariate analysis of vineyard peach (Prunus persica L. Batsch.) germplasm collection. Euphytica, 171: 227-234.

3. Hallauer, A.R. 1999. Temperate maize and heterosis. In: Coors, J. and S. Pandey (Eds.) Genetics and exploitation of heterosis in crops, CIMMYT, Mexico City 17-22 Aug. 1997 ASA, Madison, WI. pp: 353361.

4. Cantamutto, M., A. Presotto, I.F. Moroni, D. Alvarez, M. Poverene and G. Seiler. 2010. High intraspecific diversity of wild sunflowers (Helianthus annuиs L.) naturally developed in central Argentina. Flora, 205: 306-312.

5. Chungui, Q., G. Wanming, M. Jingyong, C. Xuejun, W. Xu and L. Chunzi. 1996. Relationship between genetic divergence and heterosis in sunflower. In: Proceedings of the 14th International Sunflower Conference, Beijing, Shenyang, China, 12-20 June, 1966. pp: 131-133.

6. Darvishzadeh, R. 2012. Phenotypic and molecular marker distance as a tool for prediction of heterosis and F1 performance in sunflower (Helianthus annuus L.) under well-watered and water-stressed conditions. Australian Journal of Crop Science, 6: 732-738.

7. Kholghi M., H. Hatami Maleki and R. Darvishzadeh. 2014. Diallel analysis of yield and it's related traits in sunflower (Helianthus annuus L.) under well-watered and water-stressed conditions. Agriculturae Conspectus Scientificus, 79: 175-181.

8. Fick, G.N., D.E. Zimmer and D.C. Zimmerman. 1974. Correlation of seed oil content in sunflower with other plant and seed characteristics. Crop Science, 14: 755-757.

9. Giriraj, K., T.S. Vidyashankar, M.N. Venkataram and S. Seetharam. 1979. Path coefficient analysis of seed yield in sunflower. Sunflower Newsletter, 3: 10-12.

10. Giriraj, K., N. Shivaraja and S.R. Hiremath. 1986. Studies on heterosis and inbreeding depression in selected cross combination of sunflower. Journal of Oilseeds Research, 3: 67-72.

11. Joshi, S.S., S. Ramesh, E. Gangappa, D. Jagannath and P. Chikkadevaiah. 1997. Limits to parental divergence for the occurrence of heterosis in sunflower (Helianthus annuus L.). Helia, 20: 95-100.

12. Kovačik, A. and V. Skaloud. 1978. A study of combining ability of sunflower lines and of heterosis effects on crossing them. Plant Breeding Abstracts, 48: 1040.

13. Mohan, G.S. and A. Seetharam. 2005. Genetic divergence in lines of sunflower derived from inter-specific hybridization. SABRAO Journal of Breeding and Genetics, 37: 77-84.

14. Muppidathi, N., R. Sankarapandian and S. Rajarathinam. 1995. Genetic divergence, correlation and path analysis in sunflower (Helianthus annuus L.). Crop Improvment, 22: 221-224.

15. Nooryazdan, H., H. Serieys, R. Bacilieri, J. David and A. Berville. 2010. Structure of wild annual sunflower (Helianthus annuus L.) accessions based on agro-morphological traits. Genetic Resources and Crop Evolution, 57: 27-39.

16. Presotto, A., M. Cantamutto, M. Poverene and G. Seiler. 2009. Phenotyppic diversity in wiled Helianthus annuus from Argentina. Helia, 32: 37-50.

17. Rama Subrahmanymal, S.V., S. Sudheer Kumar and A.R.G. Ranganatha. 2003. Genetic divergence for seed parameters in sunflower (Helianthus annuus L.). Helia, 26: 73-80.

18. Seetharam, A., P. Kusuma Kumari, N.M. Patil and S.S. Sindagi. 1977. Performance of hybrids of sunflower produced by means of cytoplasm male sterility. SABRAO Journal of Breeding and Genetics, 9: 51-55.

19. Skorić, D. 1974. Correlation among the most important characters of sunflower in F1 generation. In: Proceedings of 6th International Sunflower Conference, Bucharest, Romania, pp: 283-289.

20. Kholghi, M., I. Bernousi, R. Darvishzadeh, A. Pirzad and H. Hatami. 2011. Collection, evaluation and classification of Iranian confectionary sunflower (Helianthus annuus L.) populations using multivariate statistical techniques. African Journal of Biotechnology, 10: 5444-5451.

21. Smith, S.E., A.L. A1-Dos and M. Warburton. 1991. Morphological and agronomic variation in North African and Arabian alfalfa. Crop Science, 31: 1159-1163.

22. Mohammadi, S.A. and B.M. Prasanna. 2003. Analysis of genetic diversity in crop plants-salient statistical tools and considerations. Crop Science, 43: 1235-1248.

23. Cross, R.J. 1992. A proposed revision of the IBPGR barley descriptor list. Theoretical and Applied Genetics, 84: 501-507.

24. Ayana, A. and E. Becele. 1999. Multivariate analysis of morphological variation in sorghum (Sorghum bicolor L. Moench) germplasm from Ethiopia and Eritrea. Genetic Resources and Crop Evolution, 46: 273-284.

25. Hailu, F., A. Merker, H. Singh, G. Belay and E. Johansson. 2006. Multivariate analysis of diversity of tetraploid wheat germplasm from Ethiopia. Genetic Resources and Crop Evolution, 54: 83-97.

26. Upadhyaya, H.D., L.J. Reddy, S.L. Dwivedi, C.L.L. Gowda and S. Singh. 2009. Phenotypic diversity in cold-tolerant peanut (Arachis hypogaea L.) germplasm. Euphytica, 165: 279-291.

27. Hatami Maleki, H., Gh. Karimzadeh, R. Darvishzadeh and R. Alavi. 2012. Genetic diversity in oriental tobacco (Nicotiana tabacum L.) by using Multivariate statistical techniques. Iranian Journal of Field Crops Research, 10: 100-106 (In Persian). 
28. Shapiro, S.S. and M.B. Wilk. 1965. An analysis of variance test for normality (Complete samples). Biometrics, 52: 591-611.

29. Ward, J.H.Jr. 1963. Hierarchical grouping to optimize an objective function. Journal of the American Statistical Association, 58: 236-244.

30. Jobson, J.D. 1992. Applied multivariate data analysis. Volume II: Categorical and Multivariate Methods. New York: Springer-Verlag, USA.

31. Levene, H. 1960. Robust testes for equality of variances. In Contributions to Probability and Statistic. Stanford Univ. Press, Palo Alto, CA. pp: 278-292.

32. Panthee, D., R. Kc, H. Regmi, P. Subedi, S. Bhattarai and J. Dhakal. 2006. Diversity analysis of garlic (Allium sativum L.) germplasms available in Nepal based on morphological characters. Genetic Resources and Crop Evolution, 53: 205-212.

33. Lotti, C., A.R. Marcotrigiano, C. De Giovanni, P. Resta, A. Ricciardi, V. Zonno, G. Fanizza and L. Ricciardi. 2008. Univariate and multivariate analysis performed on bio-agronomical traits of Cucumis melo L. germplasm. Genetic Resources and Crop Evolution, 55: 511-522.

34. Ntundu, W.H., S.A. Shillah, W.Y.F. Marandu and J.L. Christiansen. 2006. Morphological diversity of Bambara groundnut (Vigna subterranea (L.) Verdc.) Landraces in Tanzania. Genetic Resources and Crop Evolution, 53: 367-378.

35. Dalkani, M., A. Hassani and R. Darvishzadeh. 2012. Determination of the genetic variation in Ajowan (Carum Copticum L.) populations using multivariate statistical techniques. Revista Ciência Agronômica, 43: 698-705.

36. Farahani, E. and A. Arzani. 2008. Evaluation of genetic variation of durum wheat genotypes using multivariate analysis. Electronic Journal of Crop Production, 1: 51-64 (In Persian).

37. Iglesias, L.A. and L. Iglesias. 1999. Classification of performance of wheat varieties in Cuba by means of the principal component analysis method. Cultivos Tropicales, 16: 66-69.

38. Pirkhezri, M., M.E. Hassani and M. Fakhre Tabatabai. 2009. Evaluation of genetic diversity of some German chamomile populations (Matricaria chamomilla L.) using some morphological and agronomical characteristics. Iranian Journal of Horticultural Sciences, 22: 87-99 (In Persian).

39. Sankarapandian, R., N. Muppidathi, S. Rajarathinam and S. Chdambaram. 1996. Genetic divergence in sunflower. Madras Agricultural Journal, 83: 637-639.

40. Teklewold, A., H. Jayaramaiah and G. Jayarama. 2000. Genetic divergence study in sunflower. Helia, 23: 93-104.

41. Murthy, B.R. and V. Arunachalam. 1966. The nature of genetic divergence in relation to breeding system in crop plants. Indian Journal of Genetics and Plant Breeding, 26: 188-189.

42. Stuber, C.W. 1994. Heterosis in plant breeding. Plant Breeding Reviews, 12: 227-251.

43. Hamza, S., W.B. Hamida, A. Rebai and M. Harrabi. 2004. SSR-based genetic diversity assessment among Tunisian winter barley and relationship with morphological traits. Euphytica, 135: 107-118.

44. Afkar, S., Gh. Karimzadeh and A.A. Jafari. 2009. A study of morphological variation in some genotypes of Festuca arundinacea using multivariate analysis. Iranian Journal of Field Crop Science, 40: 151-160 (In Persian). 


\title{
Study of Genetic Diversity of Agro-Morphological Traits in Confectionery Sunflower (Helianthus annuus L.) Populations using Multivariate Statistical Techniques
}

\author{
Roghayeh Ziaeifard ${ }^{1}$, Reza Darvishzadeh ${ }^{2}$ and Iraj Bernousi ${ }^{3}$

\begin{tabular}{c}
\hline \hline 1 and 3- Graduated M.Sc. and Associate Professor, Urmia University \\
2- Associate Professor, Urmia University, (Corresponding author: r.darvishzadeh@urmia.ac.ir) \\
Received: January 5, 2014 $\quad$ Accepted: May 18, 2014 \\
\hline \hline
\end{tabular}

\begin{abstract}
Confectionery sunflower (Helianthus annuus L.) is one of the valuable crops which less is known about its variability. Fifty sunflower populations have been gathered from different regions of Iran and evaluated for agro-morphological traits in completely randomized design with 10 repetitions under pot conditions. The twenty traits including days from planting to flowering, days from planting to physiological maturity, number of leaves, leaf length, petiole length, leaf width, plant height, stem diameter, head diameter, shoot dried weight, head dried weight, aerial part dried weight, harvest index, 100-seed weight, single seed weight, dehulled kernel weight, dehulled kernel weight to whole kernel, seed length, seed width, and seed dried weight per plant were evaluated. Analysis of variance showed that there is a meaningful difference between all traits except for single seed weight and stem diameter. The highest positive correlation (0.95) was observed between single seed weight and dehulled kernel weight and the highest negative one (-0.61) was observed between number of leaves and harvest index. Principal component analysis decreased the studied variables to six components with the cumulative variance of $81 \%$. Cluster analysis with Ward method classified them into four different groups. The maximum distance was observed between genotypes of groups 1 and 4 as well as 3 and 4 . The comparison of trait means in groups resulted from cluster analysis by Tukey's test demonstrated that the populations in the first group have the maximum values for most of the studied traits and populations in the fourth group have the minimum values and therefore they can be used for choosing appropriate parents in crossing programs.
\end{abstract}

Keywords: Confectionery Sunflower, Morphological Traits, Multivariable Analysis 